\title{
DYNAMICS OF EARLY TYPE GALAXIES
}

\author{
F. BERTOLA and M. CAPACCIOLI \\ Asiago Astrophysical Observatory, University of Padova, Italy
}

\begin{abstract}
An attempt has been made to compute the angular momenta of the early type galaxies NGC 4697 and NGC 4762. Comparison with spiral galaxies indicates that there is a correlation between angular momentum per unit mass and morphological class.
\end{abstract}

Angular momentum is a crucial parameter in understanding the processes of formation and evolution of galaxies. It is easily derived for spirals when the rotation curve and consequently the density distribution are known. The observed rotation curve of a spiral galaxy, both in optical and radio domain, results from measurements on emission lines produced by the flattest gaseous component, so that the circular velocity is directly derived.

The case of early type galaxies is much more complex from both the observational and theoretical point of view. Their rotation curves are obtained from absorption lines, which are difficult to record and to measure, as discussed elsewhere (Bertola and Capaccioli, 1975). Moreover many problems arise when computing the spatial velocity curve $\theta_{m}$ from the measured rotation curve $\bar{\theta}(x)$. In fact, while it is clearly evident that $\bar{\theta}(x)$ must be some weighted mean of the projected values of $\theta_{m}$ along the line of sight crossing the galaxy at $x$, we have not complete knowledge about the true nature of the weighting function. This latter must depend on:

(i) tridimensional velocity field geometry of the galaxy;

(ii) space distribution of the stellar populations contributing to the observed absorption lines;

(iii) detailed knowledge of the absorption within the galaxy;

(iv) meaning of what is measured for $\bar{\theta}(x)$, taking into account the effects on the shape of the absorption lines due to the velocity dispersion of the stars and to the observing equipment.

It seems convenient for a first approach to the problem to assume that the weighting function is the space density of light in the galaxy, as deduced from the observed luminosity profile. Even this approximation leaves a difficulty, requiring to extrapolate the rotation curve $\bar{\theta}(x)$ besides the last observed point (Bertola and Capaccioli, 1975).

In addition, in order to compute the angular momentum, the matter density distribution is required. Since the dynamics of an early type galaxy is dominated by the random motions of the stars, the velocity dispersions along three axes should be given. Presently even the most advanced observational techniques allow to obtain only the integrated value of the velocity dispersion in the center. Therefore, rather than using a wide sample of assumptions in deriving the density distribution, it seems here more convenient to have only one, that is the constancy of the mass-to-light ratio, with which the matter density results proportional to the light density. 
TABLE I

\begin{tabular}{llllll}
\hline & Morph.T. & $\begin{array}{l}D \\
(\mathrm{Mpc})\end{array}$ & $\begin{array}{l}\langle b / a\rangle \\
\text { adopted }\end{array}$ & $\begin{array}{l}\text { Mass } \\
\left(10^{10} M_{\odot}\right)\end{array}$ & $\begin{array}{l}\text { Ang. Mom. } \\
\left(10^{72} \mathrm{~g} \mathrm{~cm}^{2} \mathrm{~s}^{-1}\right)\end{array}$ \\
\hline NGC 4697 & E5 & 14.8 & 0.6 & $2.0 \times \kappa$ & $4.2 \times \kappa$ \\
NGC 4762 & SO & 14.8 & 0.2 & $1.9 \times \kappa$ & $10.0 \times \kappa$ \\
\hline
\end{tabular}

With this in mind, we discuss here the determination of the angular momentum for NGC 4697 and NGC 4762, typical examples of E and SO classes. These galaxies belong to a sample of four objects observed by one of us (F.B.) using the extremely fast image tube nebular spectrograph attached at the Cassegrain focus of the $200^{\prime \prime}$ telescope. A first account of this material has been given by Bertola (1972). The main informations about NGC 4697 and NGC 4762 are summarized in Table I.

In order to compare these early type galaxies with spirals, we computed their masses and angular momenta up to a distance from center $\bar{x}=250^{\prime \prime}(\sim 18 \mathrm{kpc})$. The

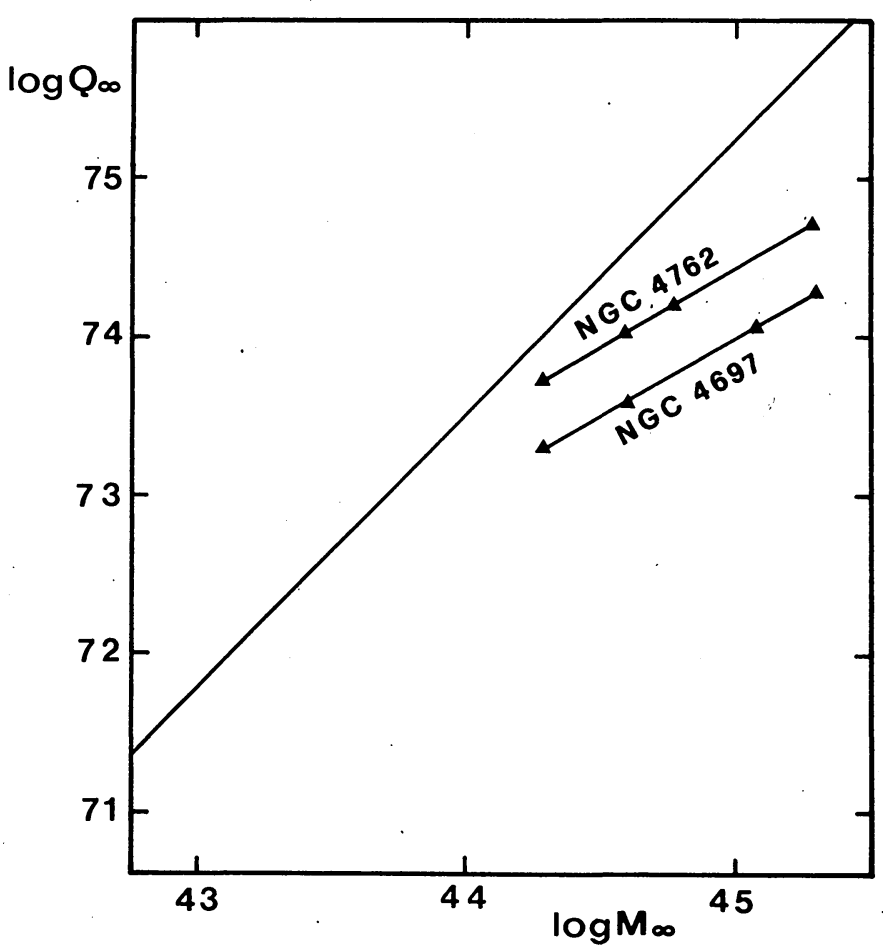

Fig. 1. The straight line represents the mass-angular momentum relation holding for spiral galaxies, from Takase and Kinoshita (1967). Segments refer to the early type galaxies NGC 4697 and NGC 4762, in the range of values of the mass-to-light ratio from 5 to 50 . Marks on segments correspond to values of the mass-to-light ratio $\kappa: 5,10,30,50$ for NGC 4697 and 5, 10, 15, 50 for NGC 4762. The elliptical NGC 4697 has a much lower angular momentum per unit mass with respect to a spiral of equal mass, while the SO lies in between. It seems likely that different mass-angular momentum relations should hold for the three morphological classes. 
conclusions we reach with this comparison are not modified by increasing $\bar{x}$. Using the observed velocity curves, together with the luminosity profiles $I(x)$ in the B system (Bertola and Capaccioli, 1975; van Houten, 1961) we derived the rotational velocities for the two galaxies. It was assumed, as a working hypothesis, that $\bar{\theta}(x)$, outside the farthest point reached by our observations, is at a constant level equal to that of the last determined velocity. Masses and angular momenta have been computed assuming a zero-thickness model characterized by a surface density $\kappa I(x)$, leaving the mass-to-light ratio $\kappa$ as a free parameter.

In Figure 1, where the mass is plotted against the angular momentum, we compare NGC 4697 and NGC 4762 with the spirals represented by the relation (straight line) found by Takase and Kinoshita (1967). Representative points for NGC 4697 and NGC 4762 lie on small segments, since $\kappa$ is assumed to vary in the range from 5 to 50 . Typical values of the mass-to-light ratios for these two galaxies could be 30 and 15 respectively. Figure 1 shows that the angular momentum per unit mass of the elliptical galaxy NGC 4697 is lower than that of a spiral of equal mass by a factor from 5 to 30 in the range of $\kappa$ considered, the most probable value being 20 . The SO galaxy NGC 4762 lies in between (factor from 2 to 10, most probable factor 5). Similar computations, carried out on the SO galaxy NGC 4111 (Capaccioli, 1974), give a result close to that on NGC 4762.

The above analysis, although based on very few cases, leads to consider the exhistence of different mass-angular momentum relations for the three classes of spiral, SO and elliptical galaxies. If this result is confirmed by further observations, it will greatly help the understanding of the processes of formation and evolution of galaxies.

\section{References}

Bertola, F.: 1972, Atti XV Riunione SAIt, 199.

Bertola, F. and Capaccioli, M.: 1975, Astrophys. J., in press.

Capaccioli, M.: 1974, Atti Cel. V Cent. Coper., Univ. Padova, 109.

Houten, C. J. van: 1961, Bull. Astron. Inst. Neth. 16, 1.

Takase, B. and Kinoshita, H.: 1967, Publ. Astron. Soc. Japan 19, 131.

\section{DISCUSSION}

Gott: The peak in Bertola's rotation curve is about $65 \mathrm{~km} \mathrm{~s}^{-1}$ while the central velocity dispersion is $310 \mathrm{~km} \mathrm{~s}^{-1}$. The isophotes at this point are still noticeably elliptical. Reasonable dynamical models would predict the rotation to be more nearly comparable with the velocity dispersion. Previous velocity dispersions by Minkowski have been shown to be in error by a factors of two. Certainly it would be advantageous to have more data both on the velocity dispersion and the rotation curve.

Bertola: I exclude that the velocity of rotation in NGC 4697 can reach a value comparable with that measured for the velocity dispersion, unless the rotation curve increases again outside the observed region.

King: Minkowski's larger velocity dispersions seem to require very little correction, in so far as we have been able to check them. It appears that only his smaller values need serious corrections. 